\title{
Filamentary structures and compact objects in the Aquila and Polaris clouds observed by Herschel ${ }^{\star, \star \star}$
}

\author{
A. Men’shchikov ${ }^{1}$, Ph. André1 ${ }^{\text {, P. Didelon }}{ }^{1}$, V. Könyves ${ }^{1}$, N. Schneider ${ }^{1}$, F. Motte $^{1}$, S. Bontemps $^{1}$, D. Arzoumanian ${ }^{1}$, \\ M. Attard ${ }^{1}$, A. Abergel ${ }^{2}$, J.-P. Baluteau ${ }^{3}$, J.-Ph. Bernard ${ }^{5}$, L. Cambrésy ${ }^{6}$, P. Cox $^{7}$, J. Di Francesco ${ }^{8}$, A. M. di Giorgio ${ }^{9}$, \\ M. Griffin ${ }^{4}$, P. Hargrave ${ }^{4}$, M. Huang ${ }^{10}$, J. Kirk ${ }^{4}$, J. Z. Li ${ }^{10}$, P. Martin ${ }^{11}$, V. Minier ${ }^{1}$, M.-A. Miville-Deschênes ${ }^{2,11}$, \\ S. Molinari ${ }^{9}$, G. Olofsson ${ }^{12}$, S. Pezzuto ${ }^{9}$, H. Roussel $^{13}$, D. Russeil ${ }^{4}$, P. Saraceno ${ }^{9}$, M. Sauvage ${ }^{1}$, B. Sibthorpe ${ }^{14}$, \\ L. Spinoglio ${ }^{9}$, L. Testi ${ }^{15}$, D. Ward-Thompson ${ }^{4}$, G. White ${ }^{16,17}$, C. D. Wilson ${ }^{18}$, A. Woodcraft ${ }^{19}$, and A. Zavagno ${ }^{4}$ \\ (Affiliations are available in the online edition)
}

Received 31 March 2010 / Accepted 3 May 2010

\section{ABSTRACT}

\begin{abstract}
Our PACS and SPIRE images of the Aquila Rift and part of the Polaris Flare regions, taken during the science demonstration phase of Herschel discovered fascinating, omnipresent filamentary structures that appear to be physically related to compact cores. We briefly describe a new multiscale, multi-wavelength source extraction method used to detect objects and measure their parameters in our Herschel images. All of the extracted starless cores (541 in Aquila and 302 in Polaris) appear to form in the long and very narrow filaments. With its combination of the far-IR resolution and sensitivity, Herschel directly reveals the filaments in which the dense cores are embedded; the filaments are resolved and have deconvolved widths of $\sim 35^{\prime \prime}$ in Aquila and $\sim 59^{\prime \prime}$ in Polaris ( 9000 AU in both regions). Our first results of observations with Herschel enable us to suggest that in general dense cores may originate in a process of fragmentation of complex networks of long, thin filaments, likely formed as a result of an interplay between gravity, interstellar turbulence, and magnetic fields. To unravel the roles of the processes, one has to obtain additional kinematic and polarization information; these follow-up observations are planned.
\end{abstract}

Key words. stars: formation - circumstellar matter - ISM: clouds - ISM: structure - infrared: ISM - submillimeter: ISM

\section{Introduction}

Herschel (Pilbratt et al. 2010) provides the best opportunity to study the earliest stages of star formation and the origin of the stellar initial mass function (IMF). Prestellar cores and young (Class 0) protostars emit the bulk of their luminosities at wavelengths 80-400 $\mu \mathrm{m}$, which makes the Herschel imaging instruments PACS (Poglitsch et al. 2010) and SPIRE (Griffin et al. 2010) perfect for performing a census of these objects down to $0.01-0.1 M_{\odot}$ in the nearby (distances $D \lesssim 500 \mathrm{pc}$ ) molecular cloud complexes. Our Herschel key program (Gould Belt Survey, André et al. 2010) aims at probing the link between diffuse cirrus-like structures and compact cores with the main scientific goal to elucidate the physical mechanisms of the formation of prestellar cores out of the diffuse medium, which is crucial for understanding the origin of stellar masses.

During the science demonstration phase of Herschel observations we imaged two large fields, the Aquila rift and part of the Polaris Flare (hereafter called Aquila and Polaris fields for brevity), with the aim of better understanding star formation in extremely different environments. The high-quality farinfrared images are extremely rich in new information on the earliest phases of star formation, which will be explored in full detail in the future. In this short paper we focus on merely one

* Herschel is an ESA space observatory with science instruments provided by European-led Principal Investigator consortia and with important participation from NASA.

$\star \star$ Figure 2 and Appendix A are only available in electronic form at http://www . aanda. org discovery made by Herschel: the impressive, ubiquitous network of filamentary structures in the interstellar medium (ISM) and its remarkably close physical relationship with the objects that appear to form within the filaments.

\section{Observations and data reduction}

The PACS instrument is described by Poglitsch et al. (2010); the SPIRE instrument, its in-orbit performance, and its scientific capabilities are described by Griffin et al. (2010), and the SPIRE astronomical calibration methods and accuracy are outlined by Swinyard et al. (2010). Our Herschel observations of the Aquila field are presented by Könyves et al. (2010); we describe here our observations of the Polaris field.

The entire Polaris field (8 square degrees) was observed on 2009 October 23, at 70,160, 250,350, and $500 \mu \mathrm{m}$ in the PACS/SPIRE parallel mode at $60^{\prime \prime} \mathrm{s}^{-1}$, performing cross-linked scans in two orthogonal directions. The PACS raw data were reduced with the HIPE 3.0.455 software with standard steps of the default pipeline. The baseline was subtracted by high-pass filtering with $\mathrm{a} \sim 1^{\circ}$ median filter width (the full leg length was 2.5 ). Multi-resolution median transform deglitching and second order deglitching were applied, and final maps were computed by the HIPE's MADmap method. The SPIRE parallel-mode data were reduced with HIPE 2.0 using pipeline scripts delivered with that version, modified to include data that were taken during the telescope's turnaround at the scan ends. A median baseline was applied to the maps on individual scan legs and a "naive mapper" was used to create images. The images were projected to the nominal 3'.2, 3'.2, and 6.'4 pixels for PACS and 6", 10", and 
$14^{\prime \prime}$ pixels for SPIRE wavelengths. In our analysis, we adopted circular Gaussian point-spread functions (PSFs) of 8.'4, 9.'4, $13^{\prime \prime} .5,18^{\prime \prime} 1,25^{\prime \prime} .2$, and $36^{\prime \prime} 9$ at the wavelengths (although the beams are actually somewhat elongated). Before extracting objects, all images were resampled to the same 6" pixel size using SWarp (Bertin et al. 2002).

\section{Source extraction method}

Here, we summarize the multi-scale, multi-wavelength source extraction algorithm that has been developed and extensively tested by A. M. at CEA Saclay. The main motivation for the development was the absence of any multi-wavelength extraction technique for use in our Herschel projects. Full description of the method and code (called getsources) as well as of the benchmarking results for this and several other extraction techniques ${ }^{1}$ will be given elsewhere (Men'shchikov et al., in prep.).

The main idea of the method used in this work is to analyze decompositions of original images (at each wavelength) across a wide range of spatial scales separated by only a small amount (typically $\sim 5 \%$ ). Each of those "single scales" are cleaned of noise and background by iterating to appropriate cut-off levels, then re-normalized and summed up over all wavelengths in a combined single-scale detection image. The main advantage of this algorithm is in its multi-wavelength design: the same combined detection image across all wavelengths eliminates the need of matching multiple catalogs obtained with different angular resolutions and reduces associated measurement errors. Besides, fine spatial decomposition improves the detection of even the faintest objects and aids in better deblending of crowded regions.

The decomposition is done by convolving the original images with circular Gaussians and subtracting them from one another (this can be called successive unsharp masking):

$I_{j}(\lambda)=G_{j-1} * I(\lambda)-G_{j} * I(\lambda) \quad(j=1,2, \ldots, N)$,

where $I(\lambda)$ is the original image at a wavelength $\lambda, I_{j}(\lambda)$ are "single-scale" decompositions, $G_{j}$ are the smoothing Gaussians ( $G_{0}$ is a two-dimensional delta function) with PSF $\lesssim F W H M \lesssim$ image size, $F W H M_{j}=f_{\mathrm{s}} \times F W H M_{j-1}, f_{\mathrm{s}}>1$ is a scale factor (usually $f_{\mathrm{s}} \approx 1.05$ ), PSF is the smallest beam over all $\lambda$, and the number of scales $N$ depends on the value of $f_{\mathrm{s}}$.

To separate the signals of objects from the noise and background contributions, we iterate to $5 \sigma_{j}(\lambda)$ cut-off levels in each of $I_{j}(\lambda)$ (the standard deviation $\sigma_{j}(\lambda)$ is computed outside the objects) and zero out all pixels with intensities below that level, producing clean images $I_{j}^{\mathrm{c}}(\lambda)$. An advantage of this single-scale cleaning is that the noise contribution depends very significantly on the scale (e.g., at large scales the small-scale noise is diluted, whereas large objects are best visible). In effect, in a full clean image $I^{\mathrm{c}}(\lambda)=\sum_{j} I_{j}^{\mathrm{c}}(\lambda)$ one would see structures deeper than in $I(\lambda)$. The above algorithm is applied to images at each band separately and the clean images are used to produce combined single-scale detection images $I_{j}^{\mathrm{c}}=\sum_{\lambda} f \times I_{j}^{\mathrm{c}}(\lambda)$ after normalization to a similar maximum intensity ${ }^{2}$. Objects in the

\footnotetext{
${ }^{1}$ The Gould Belt consortium is testing several source extraction algorithms, such as gaussclumps (Stutzki \& Guesten 1990), clumpfind (Williams et al. 1994), sextractor (Bertin \& Arnouts 1996), derivatives (Molinari et al. 2010), and a few others (see Appendix A for a very brief summary), using simulated skies of various degrees of complexity.

2 At this detection step, it is neither possible nor necessary to properly preserve the spectral behavior of sources at single scales in the combined detection image, as the processing is done at the entire image level before finding objects; spectral properties of the latter will be determined from the original images at the measurement step.
}

combined detection image are identified by tracking their appearance and "evolution" from small to large scales, and their footprints are determined. The objects are backgroundsubtracted and deblended, and their sizes and fluxes are measured in the original observed images at each wavelength. For more details, we refer interested readers to Appendix A.

\section{Results and discussion}

To derive properties of the compact objects in the Aquila and Polaris fields, we performed source extraction with getsources (Sect. 3) and made a careful analysis of the resulting extraction catalogs, selecting most reliable objects and determining core mass function for both fields (Könyves et al. 2010; André et al. 2010). To assess completeness levels of our observations, we created the synthetic skies with the actual observed background of our images (after removing all extracted objects) and populated them with cores and protostars from spherical radiative transfer models (Men'shchikov et al., in prep.), then performed extractions on the skies.

The filamentary structures are clearly visible in the left panels of Fig. 1, where both the Aquila and Polaris fields are shown as 3-color composites created from the images at 500, 160, and $70 \mu \mathrm{m}$. With red color, the images highlight strong density enhancements and low temperatures, whereas the material within two bright hot regions (HII region W 40 in the north and around MWC 297 and Sh 2-62 stellar group in the south) of the Aquila field is visible in green, blue, or white. From this one could immediately conclude where to look for prestellar cores, and it is indeed the red filaments where the reddest cores are visible. The colors are not very pronounced in the Polaris field, suggesting much smaller temperature and density variations across the field, even in its densest filaments. To better visualize and characterize the filaments, we utilized several approaches.

High-contrast images of the cores and filaments in both fields were obtained as part of our source extraction technique by applying the successive unsharp masking (Eq. (1)) with $f_{\mathrm{s}}=1.05$, $G_{1}=9$.'39, and $N=99$. In particular, the right panels in Fig. 1 show that the visibility of the very narrow and long filaments is dramatically improved in some of our "single-scale" images (which effectively select a narrow range of scales, with both larger and smaller scales filtered out). For the sake of illustration, a $40^{\prime \prime}$ scale (in which the structures are best visible) is displayed in Fig. 1. The compact objects are populating the filaments almost like pearls on threads in a necklace, giving them a nodular, lumpy appearance. The same decomposition at all Herschel wavelengths proved that the same structures are seen across the entire range, from 70 to $500 \mu \mathrm{m}$. There are, however, noticeable changes in the appearance of the filaments in Aquila (around W 40) at the shortest wavelengths (Fig. 2, upper left), presumably due to increasing opacity and to changes in temperature of some of the filaments (complex three-dimensional structures).

We also extracted filamentary structure from the observed images with the MCA software (morphological component analysis, Starck et al. 2004). The idea of the method is to morphologically decompose a signal into its building blocks, which can be represented by isotropic wavelets, ridgelets, or curvelets. Filamentary structures were separated from the more isotropic features by applying both the curvelet and wavelet transforms in 100 iterations (with exponentially decreasing thresholds) to achieve convergence in both the wavelet and curvelet representations of the original images. The curvelet component (Fig. 2, right panels; see also André et al. 2010) shows filaments very similar to those displayed in Fig. 1 and left panels of Fig. 2; we 

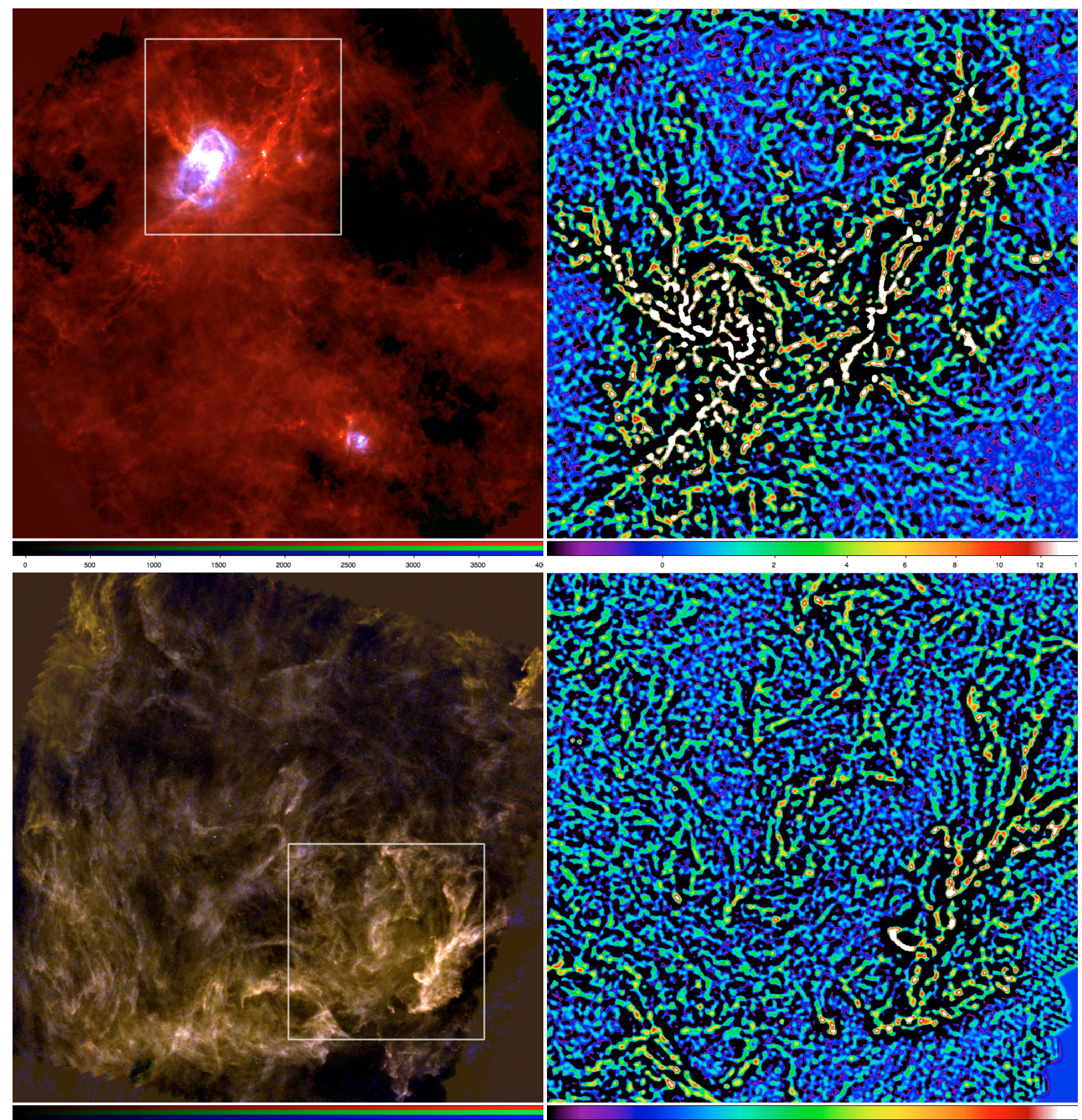

Fig. 1. Composite 3-color images (left) produced from the observed images of the Aquila (top) and Polaris (bottom) fields (panels are $3.3 \times 3.3$, or $\sim 15 \mathrm{pc}$ and $\sim 9 \mathrm{pc}$ at their distances of $260 \mathrm{pc}$ and $150 \mathrm{pc}$, respectively; see Sect. 4); the red color (SPIRE band at $350 \mu \mathrm{m}$ ), highlights colder areas and objects, whereas the green and blue colors (PACS bands at $160 \mu \mathrm{m}$ and $70 \mu \mathrm{m}$ ) show regions with progressively hotter radiation fields. High-contrast "single-scale" decompositions (right) of sub-fields in Aquila (top) and Polaris (bottom) combined from those in all SPIRE bands for better visibility, display intensity distribution (MJy/sr) within a narrow range of spatial scales around $40^{\prime \prime}$; the sub-fields of $1.2 \times 1.2$ are outlined by white squares in the left panels. Clearly visible is a very tight association of the narrow filaments with compact cores.

also get analogous results when processing the column density images (the latter are shown in Könyves et al. 2010).

Measurements of the thickness of several high-contrast filaments in our column density maps (obtained with a uniform $39^{\prime \prime}$ resolution of the $500 \mu \mathrm{m}$ band) give $48^{\prime \prime}$ for Aquila and 66" for Polaris $(F W H M)$, uncertain to within $12^{\prime \prime}$; when deconvolved, they are $28^{\prime \prime}$ and $53^{\prime \prime}$. If we adopt the distances to the regions of 260 pc (Straižys et al. 2003; Bontemps et al. 2010) and $150 \mathrm{pc}$ (Heithausen \& Thaddeus 1990), the deconvolved linear widths are $\sim 7300 \mathrm{AU}$ and $8000 \mathrm{AU}$. Measurements of the widths at several locations across a few well-behaved filaments (those not blended with the other filaments) in the observed images resulted in the values of 30,36, 42, 42, 54" (for Aquila at the PACS+SPIRE wavelengths) and 63, 64, 69" (for Polaris 
at the SPIRE wavelengths). The filaments are more resolved at PACS wavelengths and the values would imply average deconvolved widths of $35^{\prime \prime}$ for Aquila and 59" for Polaris (9000 AU in both fields). The largest FWHM sizes of the extracted cores are comparable to the filaments' widths (within $\sim 50 \%$ ) for both fields, confirming the visual impression that the objects are largely confined to the filaments they are embedded in. There seems to be a wide distribution of the lengths of the filaments; some of them are as long as $\sim 0.5$ (Figs. 1,2), which would correspond to a few pc. It is clear, however, that one needs to perform a more systematic and complete study of the filaments' properties; that will be a subject of a future work.

The Aquila and Polaris fields present very different physical conditions; the former shows very active star formation, while the latter displays almost none. Indeed, we find many extremely cold and dense filaments $\left(T_{\mathrm{d}} \approx 7.5-15 \mathrm{~K}, N_{\mathrm{H}_{2}} \approx 5 \times\right.$ $10^{20}-1.4 \times 10^{23} \mathrm{~cm}^{-2}$ ) in Aquila; most of them are gravitationally unstable and fragmented into several hundreds of prestellar cores (André et al. 2010). In contrast, the Polaris field contains warmer filaments with much lower column densities $\left(T_{\mathrm{d}} \approx\right.$ $\left.10-15 \mathrm{~K}, N_{\mathrm{H}_{2}} \approx 3 \times 10^{20}-8.6 \times 10^{21} \mathrm{~cm}^{-2}\right)$ and we find no clear examples of prestellar cores (André et al. 2010; Ward-Thompson et al. 2010). To illustrate the very close relationship between the filaments and detected objects, we overplot locations of the latter on the curvelet transform for both fields. An inspection of Fig. 2 shows that all objects detected in Aquila and Polaris are indeed located within very narrow filaments. Our maps of a stability parameter of the filaments (their mass per unit length) suggest that the Polaris filaments are stable, while many dense filaments in Aquila are gravitationally unstable (André et al. 2010).

Several possible models for the formation of filamentary cloud structures have been proposed in the literature. In particular, numerical simulations of supersonic magnetohydrodynamic turbulence in weakly magnetized clouds always generate complex systems of shocks, which fragment the gas into highdensity sheets, filaments, and cores (e.g. Padoan et al. 2001). Filamentary structures are also produced in turbulent simulations of more strongly magnetized molecular clouds, including the effects of ambipolar diffusion (Nakamura \& Li 2008). Another possibility is that the filaments originate from gravitational fragmentation of sheet-like cloud layers (Nagai et al. 1998). Interestingly, filaments can result from purely gravitational amplification of a random field of initial density fluctuations as is the case in large cosmological simulations (e.g. Ocvirk et al. 2008). Our measurements of the radial profiles of selected filaments in Aquila and Polaris suggest that magnetic fields may play an important role in shaping the structure of the observed filaments, if not in forming the filaments. Indeed, they indicate that the density falls off approximately as $\rho \propto r^{-1.5}$ (in Aquila) or $\rho \propto r^{-2}$ (in Polaris) away from the axes of the filaments. In both cases, the measured radial density profiles are much shallower than the steep $\rho \propto r^{-4}$ profile of unmagnetized hydrostatic filaments (Ostriker 1964), as already pointed out by Lada et al. (1999) and Johnstone \& Bally (1999) for two specific filaments. Models of unmagnetized equilibrium filaments are therefore inconsistent with observations ${ }^{3}$. By contrast, models of equilibrium filaments with primarily toroidal or helical magnetic fields can account for radial profiles ranging from $\rho \propto r^{-1}$ to $\rho \propto r^{-2}$, in agreement with observations (Fiege \& Pudritz 2000).

\footnotetext{
3 Unmagnetized, non-equilibrium filaments embedded in sheet-like structures can however have shallow profiles consistent with observations (S. Inutsuka, private communication).
}

Filaments are seen in numerous astronomical images and the filamentary structure of molecular clouds has been known for some time (e.g. Schneider \& Elmegreen 1979; Goldsmith et al. 2008). However, Herschel with its combination of the far-IR resolution and sensitivity directly reveals the filaments in which the cores are forming. Our first observations demonstrate the fascinating, omnipresent filamentary density structure of the cold ISM and its extraordinarily intimate physical relationship with the objects that form within the filaments. They enable us to suggest that in general dense cores may originate in a process of fragmentation of long, thin filaments, presumably formed as a result of an interplay between gravity, interstellar turbulence, and magnetic fields (see also discussion in André et al. 2010). To unravel the role and relative importance of these processes, one needs additional kinematic and polarization information (cf. Matthews et al. 2001); these follow-up observations are planned.

Acknowledgements. PACS has been developed by a consortium of institutes led by MPE (Germany) and including UVIE (Austria); KU Leuven, CSL, IMEC (Belgium); CEA, LAM (France); MPIA (Germany); INAF-IFSI/ OAA/OAP/OAT, LENS, SISSA (Italy); IAC (Spain). This development has been supported by the funding agencies BMVIT (Austria), ESA-PRODEX (Belgium), CEA/CNES (France), DLR (Germany), ASI/INAF (Italy), and CICYT/MCYT (Spain). SPIRE has been developed by a consortium of institutes led by Cardiff Univ. (UK) and including Univ. Lethbridge (Canada); NAOC (China); CEA, LAM (France); IFSI, Univ. Padua (Italy); IAC (Spain); Stockholm Observatory (Sweden); Imperial College London, RAL, UCL-MSSL, UKATC, Univ. Sussex (UK); Caltech, JPL, NHSC, Univ. Colorado (USA). This development has been supported by national funding agencies: CSA (Canada); NAOC (China); CEA, CNES, CNRS (France); ASI (Italy); MCINN (Spain); SNSB (Sweden); STFC (UK); and NASA (USA).

\section{References}

André, Ph., Men'shchikov, A., Bontemps, S., et al. 2010, A\&A, 518, L102 Bertin, E., \& Arnouts, S. 1996, A\&AS, 117, 393

Bertin, E., Mellier, Y., Radovich, M., et al. 2002, in Astronomical Data Analysis Software and Systems XI, ed. D. A. Bohlender, D. Durand, \& T. H. Handley, ASP Conf. Ser., 281, 228

Bontemps, S., André, Ph., Könyves, V., et al. 2010, A\&A, 518, L85 Fiege, J. D., \& Pudritz, R. E. 2000, MNRAS, 311, 85 Goldsmith, P. F., Heyer, M., Narayanan, G., et al. 2008, ApJ, 680, 428 Griffin, M. J., Abergel, A., Abreu, A., et al. 2010, A\&A, 518, L3 Heithausen, A., \& Thaddeus, P. 1990, ApJ, 353, L49 Johnstone, D., \& Bally, J. 1999, ApJ, 510, L49

Könyves, V., André, Ph., Men'shchikov, A., et al. 2010, A\&A, 518, L106 Lada, C. J., Alves, J., \& Lada, E. A. 1999, ApJ, 512, 250

Matthews, B. C., Wilson, C. D., \& Fiege, J. D. 2001, ApJ, 562, 400 Moffat, A. F. J. 1969, A\&A, 3, 455

Molinari, S., Swinyard, B., Bally, J., et al. 2010, A\&A, 518, L100

Motte, F., Bontemps, S., Schilke, P., et al. 2007, A\&A, 476, 1243

Nagai, T., Inutsuka, S.-I., \& Miyama, S. M. 1998, ApJ, 506, 306 Nakamura, F., \& Li, Z.-Y. 2008, ApJ, 687, 354

Ocvirk, P., Pichon, C., \& Teyssier, R. 2008, MNRAS, 390, 1326 Ostriker, J. 1964, ApJ, 140, 1056

Padoan, P., Juvela, M., Goodman, A. A., \& Nordlund, Å. 2001, ApJ, 553, 227 Pilbratt, G. L., Riedinger, J. R., Passvogel, T., et al. 2010, A\&A, 518, L1 Poglitsch, A., Waelkens, C., Geis, N., et al. 2010, A\&A, 518, L2 Schneider, S., \& Elmegreen, B. G. 1979, ApJS, 41, 87

Smith, A. R. 1979, in SIGGRAPH'79: Proc. of the 6th annual conference on Computer graphics and interactive techniques (New York: ACM), 276

Starck, J.-L., \& Murtagh, F. 2006, Astronomical Image and Data Analysis, ed. J.-L. Starck, \& F. Murtagh

Starck, J.-L., Elad, M., \& Donoho, D. L. 2004, in Advances in Imaging and Electron Physics, 132

Straižys, V., Černis, K., \& Bartašiūtè, S. 2003, A\&A, 405, 585

Stutzki, J., \& Guesten, R. 1990, ApJ, 356, 513

Swinyard, B. M., Ade, P., Baluteau, J.-P., et al. 2010, A\&A, 518, L4 Ward-Thompson, D., Kirk, J., André, Ph., et al. 2010, A\&A, 518, L92 Williams, J. P., de Geus, E. J., \& Blitz, L. 1994, ApJ, 428, 693 
1 Laboratoire AIM, CEA/DSM-CNRS-Université Paris Diderot, IRFU/Service d'Astrophysique, C.E. Saclay, Orme des Merisiers, 91191 Gif-sur-Yvette, France e-mail: alexander.menshchikov@cea.fr

2 Institut d'Astrophysique Spatiale (CNRS), Université Paris-Sud, bât. 121, 91405 Orsay, France

3 Laboratoire d'Astrophysique de Marseille, CNRS/INSU-Université de Provence, 13388 Marseille Cedex 13, France

4 School of Physics and Astronomy, Cardiff University, Queens Buildings, The Parade, Cardiff CF24 3AA, UK

5 CESR, 9 Avenue du Colonel Roche, B.P. 4346, 31029 Toulouse, France

${ }^{6}$ CDS, Observatoire de Strasbourg, 11, Rue de l'Université, 67000 Strasbourg, France

7 IRAM, 300 Rue de la Piscine, Domaine Universitaire, 38406 SaintMartin-d'Héres, France

8 Herzberg Institute of Astrophysics, University of Victoria, Department of Physics and Astronomy, Victoria, Canada

9 INAF-IFSI, Fosso del Cavaliere 100, 00133 Roma, Italy
10 National Astronomical Observatories, Chinese Academy of Sciences, A20 Datun Road, Chaoyang District, Beijing 100012, PR China

11 Canadian Institute for Theoretical Astrophysics, University of Toronto, 60 St. George Street, Toronto, ON, M5S 3H8, Canada

12 Department of Astronomy, Stockholm University, AlbaNova University Center, 10691 Stockholm, Sweden

13 Institut d'Astrophysique de Paris, UMR7095 CNRS, Université Pierre \& Marie Curie, 98 bis Boulevard Arago, 75014 Paris, France

14 UK Astronomy Technology Centre, Royal Observatory Edinburgh, Blackford Hill, EH9 3HJ, UK

15 Istituto Nationale di Astrofisica, Largo Enrico Fermi 5, 50125 Firenze, Italy

16 The Rutherford Appleton Laboratory, Chilton, Didcot OX11 0NL, UK

17 Department of Physics \& Astronomy, The Open University, Milton Keynes MK7 6AA, UK

18 Dept. of Physics \& Astronomy, McMaster University, Hamilton, Ontario, L8S 4M1, Canada

19 UK Astronomy Technology Center, Royal Observatory Edinburgh, Edinburgh, EH9 3HJ, UK 

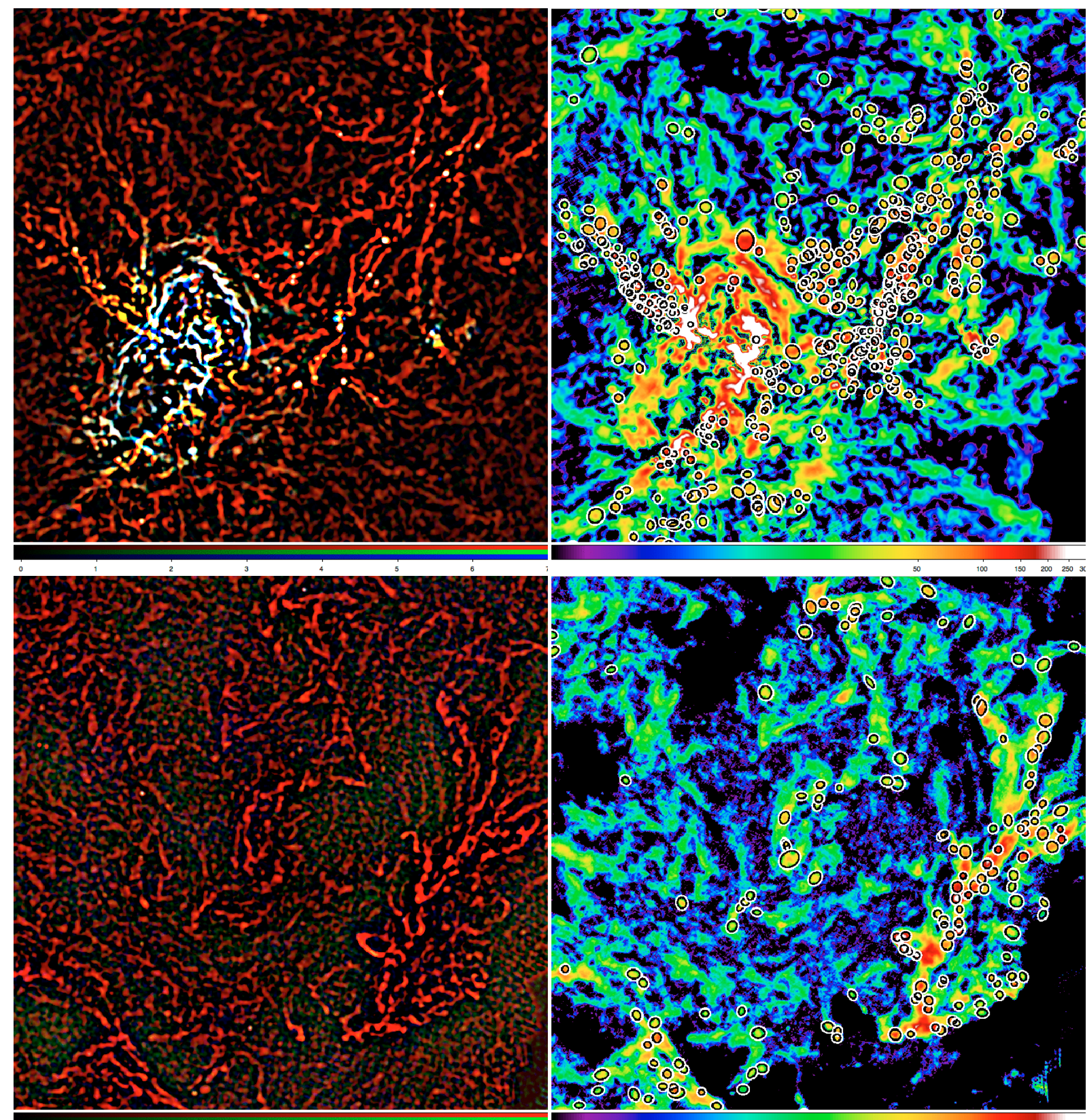

Fig. 2. Composite 3-color images left of the sub-fields of Aquila (top) and Polaris (bottom) produced from the high-contrast "single-scale" decompositions (red comes from all SPIRE bands, green and blue correspond to the PACS bands at 160 and $70 \mu \mathrm{m}$, respectively); the sub-fields and decompositions are the same as in Fig. 1. Curvelet components (right) extracted from the original SPIRE $350 \mu$ m images overlaid with ellipses for selected cores extracted by getsources (shown are starless cores: those detected at $S / N \geq 7.5$ levels in at least two SPIRE bands and not detected in emission or detected in absorption in the PACS $70 \mu \mathrm{m}$ band, with SED dust temperatures $T_{\mathrm{d}} \leq 18 \mathrm{~K}$; see also Könyves et al. 2010 ; André et al. 2010). Most objects lie in the narrow and long filaments spanning orders of magnitude in intensity (MJy/sr). 


\section{Appendix A: Extraction techniques}

\section{A.1. Existing source extraction algorithms}

Here we summarize (very briefly) the concepts of different techniques, to place getsources described in Sect. 3 in a wider context. The algorithms trying to solve the same problem of source extraction originated from different ideas. Note that they have also been developed (oriented) for use in different areas of astronomy, thus their performance for a specific project must be carefully tested before an appropriate method can be chosen.

Stutzki \& Guesten (1990)'s gaussclumps (originally created for position-velocity cubes) fits a Gaussian profile to the brightest peak, subtracting the fit from the image, then fitting a new profile to the brightest peak in the image of residuals, iterating until some termination criteria are met. Williams et al. (1994)'s clumpfind contours an image at a number of levels, starting from the brightest peak in the image and descending down to a minimum contour level, marking as clumps along the way all connected areas of pixels that are above the contour level. Bertin \& Arnouts (1996)'s sextractor estimates and subtracts background, then uses thresholding to find objects, deblends them if they overlap, and measures their positions and sizes using intensity moments. CUPID ${ }^{4}$ 's reinhold identifies pixels within the image which mark the edges of clumps of emission, producing a set of rings around the clumps. After cleaning noise effects on the edges, all pixels within each ring are assumed to belong to a single clump. CUPID's fellwalker ascends image peaks by following the line of the steepest ascent, considering every pixel in the image as a starting point for a walk to a significant peak, marking along the way all visited pixels with a clump identifier. Motte et al. (2007)'s mre-gcl combines cloud filtering techniques based on wavelet multi-resolution algorithms (e.g., Starck \& Murtagh 2006) with gaussclumps. Molinari et al. (2010)'s derivatives analyzes multi-directional second derivatives of the original image and performs curvature thresholding to isolate compact objects, then fits variable-size elliptical Gaussians (adding also a planar background) at their positions. Another method that defines cores in terms of connected pixels is csar, which was developed for use with BLAST and Herschel (Harry et al. 2010, in preparation).

Whereas clumpfind, reinhold, fellwalker, and csar merely partition the image between objects not allowing them to overlap, gaussclumps, sextractor, and mre-gcl can deblend overlapping objects, which is quite essential for obtaining correct results in crowded regions. None of the methods was designed to handle multi-wavelength data, making it necessary to match the catalogs obtained at different wavelengths using an association radius as a parameter.

\section{A.2. More details on the new method}

In getsources the extraction of objects is performed in each of the combined detection images by going from the smallest to the largest scales and finding segmentation masks of the objects at each scale using the tint fill algorithm (Smith 1979) ${ }^{5}$. The masks are the areas of connected pixels in a segmentation image, and the algorithm fills the pixels' values with the number of a detected object and allows tracking of all pixels belonging to the object across all scales. The segmentation masks expand toward larger scales, and the evolution of each object's mask is followed, as is appearance of new objects at any scale and disappearance of those which become too faint at the current and larger scales. When two or more objects touch each other in a single-scale image, the segmentation masks are not allowed to overlap, but overlapping does happen between objects of different scales. The largest extent of any source defines its footprint, and this is determined at the scale where the object's contrast above the cut-off level is at maximum. The scale itself provides an initial estimate for the object's FWHM size.

The positions of sources are computed from the first moments of intensities in a combined detection image at a range of single scales, from where an object first appeared and to the scale twice as large. The objects' sizes are computed from the first and second intensity moments in the original background-subtracted image. The background subtraction is done by linearly interpolating pixel intensities off the observed image under the footprints, in the four main directions (two axes and two diagonals), based on the pixel values just outside the footprints. Our iterative deblending algorithm employs two-dimensional shapes with peak intensities and sizes of the extracted objects in order to divide the intensity of a pixel between surrounding objects according to the fraction of the shapes' intensities at the pixel. For the shapes we adopted a two-dimensional analog of the Gaussianlike function $f_{0}\left(1+\left(r / r_{0}\right)^{2}\right)^{-\alpha}$ (Moffat 1969) with $\alpha=10$.

The end result of the processing is an extraction catalog (one line per object) containing coordinates of all detections (independent of $\lambda$ ) and estimates of the objects' $S / N$ ratios, peak and total fluxes (with their uncertainties), and sizes and orientations for each wavelength. In addition, getsources produces catalogs of all possible colors, as well as the azimuthally-averaged intensity profiles (their full, background-subtracted, and deblended versions) and deblended images for each object.

\footnotetext{
${ }^{4}$ CUPID is a source extraction software package developed by the STARLINK team for use with the SCUBA2 surveys; it is a general wrapper to which additional methods can be added. See documentation: http://docs.jach.hawaii.edu/star/sun255.htx/ sun255.html
}

\author{
5 Available at \\ http://portal.acm.org/citation. cfm?id=800249.807456
}

